\title{
Analysis of Multiple Structural Changes in Financial Contagion Based on the Largest Lyapunov Exponents
}

\author{
Rui Wang, Xiaofeng Hui, and Xuechao Zhang \\ School of Management, Harbin Institute of Technology, Yikuang Street 2, Harbin 150080, China \\ Correspondence should be addressed to Rui Wang; wangrui87@gmail.com
}

Received 11 January 2014; Accepted 30 May 2014; Published 19 June 2014

Academic Editor: Pankaj Gupta

Copyright (C) 2014 Rui Wang et al. This is an open access article distributed under the Creative Commons Attribution License, which permits unrestricted use, distribution, and reproduction in any medium, provided the original work is properly cited.

\begin{abstract}
A modified multiple structural changes model is built to test structural breaks of the financial system based on calculating the largest Lyapunov exponents of the financial time series. Afterwards, the Lorenz system is used as a simulation example to inspect the new model. As the Lorenz system has strong nonlinearity, the verification results show that the new model has good capability in both finding the breakpoint and revealing the changes in nonlinear characteristics of the time series. The empirical study based on the model used daily data from the S\&P 500 stock index during the global financial crisis from 2005 to 2012 . The results provide four breakpoints of the period, which divide the contagion into four stages: stationary, local outbreak, global outbreak, and recovery period. An additional significant result is the obvious chaos characteristic difference in the largest Lyapunov exponents and the standard deviation at various stages, particularly at the local outbreak stage.
\end{abstract}

\section{Introduction}

The frequent financial crises since the 1990s have attracted the attention of many researchers. Each crisis, for example, the ERM crisis in 1992-1993, the Mexican crisis in 1994-1995, the Asian crisis in 1997-1998, the Russian crisis in 1998, the Argentinian crisis in 2001, and the global financial crisis in 2009 , shows strong contagion effect and nonlinearity.

Many earlier studies report a strong increase in the correlations of returns between markets. Billio and Pelizzon [1] and Gravelle et al. [2] argue that a structural break in the correlations demonstrates that the international propagation mechanisms of financial shocks are discontinuous. Masson [3] studies the structural break and attributes this break to financial panic, or the herding or switching of expectations across multiple equilibria. Kristin and Rigobon [4] discuss crisis-contingent theories, qualifying this phenomenon as "shift-contagion."

Rigobon [5] studies the stability of the international propagation mechanisms between 36 stock markets during three recent international financial crises (Mexico 1994, Asia 1997, and Russia 1998). The results illustrate that the increase in the correlation between these stock markets does not result from instability in the mechanisms of propagation but rather is the consequence of a strong interdependence during the crisis periods and the stability periods. Considering the robustness of the conclusions of Rigobon [5], Billio and Pelizzon [1] find that the size of the crisis window has an important influence on the sensitivity of the results. Gravelle et al. [2] note that the subjective and arbitrary choice of the structural change points, which define the beginning and the end of the crisis window, is an important issue.

To solve this problem of the crisis window definition, Caporale et al. [6] test for the stability of the propagation mechanisms using an approach based on an estimate of the full sample. Their results suggest the existence of the contagion between the Asian stock markets. Using the same approach, McAleer and Nam [7] also verify the contagion between the Asian foreign exchange markets. However, none of these studies test for structural change in correlation across financial markets but rather test for nonlinearity of the financial interdependence model using dummy variables.

Some papers extend from the existing literature by using the recently developed structural change approach of Bai and Perron [8] to investigate the crisis contagion and to solve the crisis window definition problem. Essaadi et al. [9] test for contagion caused by the Thai baht collapse of July 1997. In line with earlier work, shift-contagion is defined as a structural 
change within the international propagation mechanisms of financial shocks. They adopt Bai and Perron's [8] structural break approach to detect the endogenous breakpoints of the pairwise, time-varying correlations between Thailand and seven Asian stock markets returns. Their approach enables us to solve the misspecification problem of the crisis window. Baek and Jun [10] test for the existence of financial contagion using a method that allows an incubation period before contagion takes effect. They define contagion as an increase in cross-market linkages following shocks. With daily data on Asian stock markets during the 1997-1998 crisis, they find significant upward shifts in the linkages between the Asian markets of both crisis and noncrisis countries. The upward shifts are maintained even after controlling for heteroskedasticity and common world and regional factors, providing strong evidence for financial contagion.

The literature on capital markets reveals some evidence of the existence of nonlinear or chaotic phenomena in financial markets starting with the contributions by Hsieh [11]. More recently, Lim and Liew [12] find substantive evidence on nonlinear dynamics in the ASEAN financial markets. The financial data at the term of crisis are often nonlinear deterministic features. The traditional theory of financial crisis, which is often based on a statistical framework, makes it impossible to correctly analyze the structure of crisis contagion. Detecting the presence of chaos in a dynamical system is an important problem that is solved by measuring the largest Lyapunov exponents. Lyapunov exponents quantify the exponential divergence of initially close state-space trajectories and estimate the amount of chaos in a system. The presence of a positive exponent is sufficient for diagnosing chaos and represents local instability in a particular direction.

In our study, the focus is on the relatively neglected fact that financial crisis may take time. In previous studies, one shared assumption is that contagion is instantaneous. However, we note that the structure of the financial system may differ in each crisis period and that structural breaks may exist. We treat the breakpoints as unknowns and use the methods of Bai and Perron $[8,13]$ to estimate them. Moreover, instead of relying on the index and correlation approach, we test for a structural break in the largest Lyapunov exponents during crisis periods.

\section{Method}

2.1. The Largest Lyapunov Exponents. Rosenstein et al. [14] present a practical method for calculating the largest Lyapunov exponents from small data sets. The method is reliable for small data sets and is fast and easy to implement.

The first step of our approach involves reconstructing the attractor dynamics from a single time series. For an $N$-point time series $\left\{x_{t}\right\}_{t=1}^{T}$,

$$
\begin{gathered}
X(t)=\left[x\left(t_{i}\right), x\left(t_{i}+\tau\right), x\left(t_{i}+2 \tau\right), \ldots, x\left(t_{i}+(m-1) \tau\right)\right], \\
i=1,2, \ldots, M, \\
M=N-(m-1) \tau,
\end{gathered}
$$

where $\tau$ is the reconstruction delay and $m$ is the embedding dimension.

After reconstructing the dynamics, the algorithm locates the nearest neighbor of each point on the trajectory. The nearest neighbor $X(\hat{t})$ is found by searching for the point that minimizes the distance to the particular reference point $X(t)$ :

$$
d_{t}(0)=\min _{\widehat{t}}\|X(t)-X(\widehat{t})\|, \quad|t-\hat{t}|>p,
$$

where $\|\cdot\|$ denotes the Euclidean norm. We impose the additional constraints that the nearest neighbors have a temporal separation greater than the mean period of the time series and that $p$ is the mean period and is estimated by the reciprocal of the mean frequency of the power spectrum. The largest Lyapunov exponent is then estimated as the mean rate of separation of the nearest neighbors.

Then, we calculate $d_{t}(i)$, the distance between the $t$ th pair of the nearest neighbors after $i$ discrete-time steps, as follows:

$$
\begin{gathered}
d_{t}(i)=\|X(t+i)-X(\widehat{t}+i)\| \\
i=1,2, \ldots, \min (M-t, M-\widehat{t}) .
\end{gathered}
$$

Finally, we estimate the mean value $y_{i}$ of $\ln d_{t}(i)$ according to $i$ and $t$ :

$$
y(i)=\frac{1}{q \Delta t} \sum_{j=1}^{q} \ln d_{j}(i),
$$

where $\Delta t$ is the sampling period of the time series and $q$ is the number of nonzero $d_{i}(i)$. The remainder of our method proceeds as the largest Lyapunov exponent is easily and accurately calculated using least squares fit to the equation above.

2.2. Multiple Structural Changes Model. Bai and Perron contribute greatly to the development of the time series multiple structural changes model, especially on the aspects of the parameters estimation, the coherence of breakpoints and confidence intervals, and the detection of breakpoints.

We consider the following $m+1$ multiple linear regression with $m$ breakpoints:

$$
\begin{gathered}
y_{t}=x_{t}^{\prime} \beta+z_{t}^{\prime} \delta_{1}+\mu_{t}, \quad t=1,2, \ldots, T_{1}, \\
y_{t}=x_{t}^{\prime} \beta+z_{t}^{\prime} \delta_{2}+\mu_{t}, \quad t=T_{1}+1, \ldots, T_{2},
\end{gathered}
$$

$$
y_{t}=x_{t}^{\prime} \beta+z_{t}^{\prime} \delta_{m+1}+\mu_{t}, \quad t=T_{m}+1, \ldots, T,
$$

where $y_{t}$ is the observed dependent variable at time $t$ and $\left(x_{t}\right)_{p \times 1}$ and $\left(z_{t}\right)_{q \times 1}$ are the vectors of covariates, while $(\beta)_{p \times 1}$ and $\left(\delta_{j}\right)_{q \times 1}(j=1,2, \ldots, m)$ are the corresponding vectors of coefficients and $\mu$ is the disturbance at time $t$. The indices $\left(T_{1}, T_{2}, \ldots, T_{m}\right)$ are the $m$ breakpoints. The associated leastsquares estimates are obtained by minimizing the sum of squared residuals:

$$
S_{T}\left(T_{1}, T_{2}, \ldots, T_{m}\right)=\sum_{j=1}^{m+1} \sum_{t=T_{j-1}+1}^{T_{j}}\left(y_{t}-x_{t}^{\prime} \beta-z_{t}^{\prime} \delta_{j}\right)^{2} .
$$


TABLE 1: Estimate results with the multiple structural changes model.

\begin{tabular}{|c|c|c|c|c|c|c|}
\hline \multicolumn{7}{|c|}{ Specifications } \\
\hline$z_{t}=\{1\}$ & $q=1$ & $p=0$ & $h=303$ & $M=5$ & $\varepsilon=0.15$ & $T=2019$ \\
\hline \multicolumn{7}{|c|}{ Tests } \\
\hline $\operatorname{SupF}_{T}(1)$ & $\operatorname{SupF}_{T}(2)$ & $\operatorname{SupF}_{T}(3)$ & $\operatorname{SupF}_{T}(4)$ & $\operatorname{SupF_{T}(5)}$ & UDmax & WDmax \\
\hline 1.4735 & $8.286^{*}$ & $11.7107^{*}$ & $18.8166^{*}$ & $13.0529^{*}$ & $18.8166^{*}$ & $18.8166^{*}$ \\
\hline $\operatorname{SupF}_{T}(2 \mid 1)$ & $\operatorname{SupF}_{T}(3 \mid 2)$ & $\operatorname{SupF}_{T}(4 \mid 3)$ & $\operatorname{SupF}_{T}(5 \mid 4)$ & & & \\
\hline $20.8615^{*}$ & $10.1503^{*}$ & 0 & 0 & & & \\
\hline \multicolumn{7}{|c|}{ Number of breaks selected } \\
\hline Number of breaks & 0 & 1 & 2 & 3 & 4 & 5 \\
\hline BIC & 10.3216 & 10.0755 & 9.3698 & 9.0284 & 8.7457 & 8.8352 \\
\hline LWZ & 10.3221 & 10.0904 & 9.3993 & 9.0723 & 8.8041 & 8.9081 \\
\hline \multicolumn{7}{|c|}{ Estimates with three breaks } \\
\hline$\widehat{\delta}$ & $\widehat{\delta}$ & $\widehat{\delta}$ & $\widehat{\delta}$ & $\widehat{\delta}$ & & \\
\hline 1239.26 & 1413.78 & 936.1 & 1147.23 & 1324.82 & & \\
\hline$(30.5036)$ & $(41.1877)$ & $(41.9042)$ & $(83.8616)$ & $(41.2039)$ & & \\
\hline$\widehat{T}$ & $\widehat{T}$ & $\widehat{T}$ & $\widehat{T}$ & & & \\
\hline $2006 / 9 / 25$ & $2008 / 9 / 12$ & $2009 / 11 / 23$ & $2011 / 2 / 1$ & & & \\
\hline
\end{tabular}

${ }^{*}$ Significance at the $5 \%$ level.

Let $\widehat{\beta}$ and $\widehat{\delta}$ denote the estimates based on the given $m$ partition $\left(T_{1}, T_{2}, \ldots, T_{m}\right)$. Substituting these in the objective function and denoting the resulting sum of squared residuals as $S_{T}\left(T_{1}, T_{2}, \ldots, T_{m}\right)$, the estimated breakpoints $\left(T_{1}, T_{2}, \ldots, T_{m}\right)$ are such that

$$
\left(\widehat{T}_{1}, \widehat{T}_{2}, \ldots, \widehat{T}_{m}\right)=\arg \left[\min S_{T}\left(T_{1}, T_{2}, \ldots, T_{m}\right)\right] .
$$

Based on the method above, Bai and Perron $[13,15]$ construct Sup $F_{T}(k, q)$, UDmax, WDmax, Sup $F_{T}(\ell+1 \mid \ell)$, BIC, and LWZ with these statistic indices to estimate the optimal number of structure breakpoints and the degree of confidence. Bai and Perron [16] also provide some values of the trimming $\varepsilon$ and a maximum possible number of breaks $M$ to the tests. In this paper, we have chosen $\varepsilon=0.15$ and $M=5$.

\subsection{Modified Multiple Structural Changes Model Based on the} Largest Lyapunov Exponents. First, we choose an appropriate time window $n n\left(n n>10^{D}\right)$, and $D$ is the dimension of strange attractor, which is calculated by the $G-P$ method $[17,18]$. Then, we calculate the largest Lyapunov exponents $\lambda_{i}$ of time window $\left(T_{i}-(n n / 2), \ldots, T_{i}-1, T_{i}, T_{i}+1, \ldots, T_{i}+\right.$ $(n n / 2)-1), i=(n n / 2)+1,(n n / 2)+2, \ldots, T-(n n / 2)$, using the method presented by Rosenstein et al. [14]. Therefore, we obtain the $(T-n n+1)$ largest Lyapunov exponents $\lambda_{i}$ of $Y=\left(y_{1}, y_{2}, \ldots, y_{T}\right)^{\prime}$ in $Y=X \beta+Z \delta+U$, and we can obtain $m$ structural breaks and the corresponding statistics.

\section{Simulation Experiment for Modified Model}

We use the Lorenz system as an example to simulate an experiment about the modified multiple structural changes model. The data contains two parts: (1) $N=1: 3000, \sigma=16$, $b=4$, and $\gamma=45.92$, and (2) $N=3001: 6000, \sigma=10$, $b=8 / 3$, and $\gamma=28$. We see the results with the original multiple structural changes model in Table 1.

We use the C-C method to reconstruct the phase spaces with the simulation experiment data and obtain the time lag $\tau=16$ and the embedding dimension $m=9$. We calculate the dimension of the attractor $D$ as 1.7092 by the $G-P$ method; therefore, the time window $n n>10^{D}=51.19$. This paper selects the window width $n n=300$. The estimate results with the modified model are shown in Table 2.

Compared with the results of Tables 2 and 3, we find that when using the original model for the simulation data to analyze the breakpoint all statistics cannot pass the tests and that the optimal two breakpoints are in 2327 and 3835 . We conclude that the original model is unable to properly identify the breakpoint of the time series. With the modified model, the Sup $F_{T}(k)$ tests and the double maximum tests UDmax and WDmax are all significant at the 5\% level. The significance of these tests means that one break is at least present. Combined with the $\operatorname{Sup}_{T}(\ell+1 \mid \ell)$ tests and criteria BIC and LWZ, we determine that the optimal number of breakpoints is one and that the break is in 3040, which is very similar with the actual situation. The results show that the modified model has very good judgment of breakpoint.

\section{Empirical Analysis}

After the simulation experiment, we use the S\&P 500 stock index during the 2008 global financial contagion to test for the modified model. The sampling time periods for empirical analysis are the 2019 daily observations from January 1, 2005, to December 31, 2012. This paper smoothens the daily closing price data with the logarithmic yield method and obtains the logarithmic yield $x_{\mathrm{ROR}}(t)$ (Figure 1):

$$
x_{\mathrm{ROR}}(t)=\ln \frac{P(t)}{P(t-1)}, \quad t=1,2, \ldots, N .
$$


TABLE 2: Estimate results with the original model.

\begin{tabular}{|c|c|c|c|c|c|c|}
\hline \multicolumn{7}{|c|}{ Specifications } \\
\hline$z_{t}=\{1\}$ & $q=1$ & $p=0$ & $h=1500$ & $M=2$ & $\varepsilon=0.25$ & $T=6000$ \\
\hline \multicolumn{7}{|c|}{ Tests } \\
\hline $\operatorname{SupF}_{T}(1)$ & $\operatorname{SupF}_{T}(2)$ & UDmax & WDmax & $\operatorname{SupF}_{T}(2 \mid 1)$ & & \\
\hline 0.1192 & 0.0404 & 0.1192 & 0.1192 & 0.02853 & & \\
\hline \multicolumn{7}{|c|}{ Number of breaks selected } \\
\hline Number of breaks & 0 & 1 & 2 & & & \\
\hline BIC & 4.7196 & 4.7113 & 4.7039 & & & \\
\hline LWZ & 4.7198 & 4.7183 & 4.7176 & & & \\
\hline \multicolumn{7}{|c|}{ Estimates with three breaks } \\
\hline$\widehat{\delta}_{1}$ & $\widehat{\delta}_{2}$ & $\widehat{\delta}_{3}$ & & & & \\
\hline-0.2678 & -0.7618 & 1.1165 & & & & \\
\hline$(4.5047)$ & $(15.7582)$ & $(4.1354)$ & & & & \\
\hline$\widehat{T}_{1}$ & $\widehat{T}_{2}$ & & & & & \\
\hline 2327 & 3835 & & & & & \\
\hline
\end{tabular}

${ }^{*}$ Significance at the $5 \%$ level.

TABLE 3: Estimate results with the modified model.

\begin{tabular}{|c|c|c|c|c|c|c|}
\hline \multicolumn{7}{|c|}{ Specifications } \\
\hline$z_{t}=\{1\}$ & $q=1$ & $p=0$ & $h=1425$ & $M=2$ & $\varepsilon=0.25$ & $T=5701$ \\
\hline \multicolumn{7}{|c|}{ Tests } \\
\hline $\operatorname{SupF}_{T}(1)$ & $\operatorname{SupF}_{T}(2)$ & $U D \max$ & WDmax & $\operatorname{SupF}_{T}(2 \mid 1)$ & & \\
\hline $13.0894^{*}$ & $6.6668^{*}$ & $13.0894^{*}$ & $13.0894^{*}$ & 0.69176 & & \\
\hline \multicolumn{7}{|c|}{ Number of breaks selected } \\
\hline Number of breaks & 0 & 1 & 2 & & & \\
\hline $\mathrm{BIC}$ & -3.0074 & -4.0827 & -4.103 & & & \\
\hline LWZ & -3.0073 & -4.0755 & -4.0887 & & & \\
\hline \multicolumn{7}{|c|}{ Estimates with three breaks } \\
\hline$\widehat{\delta}_{1}$ & $\widehat{\delta}_{2}$ & & & & & \\
\hline-0.3058 & 0.5321 & & & & & \\
\hline$(0.038091)$ & $(0.092262)$ & & & & & \\
\hline \multicolumn{7}{|l|}{$\widehat{T}_{1}$} \\
\hline 3040 & & & & & & \\
\hline
\end{tabular}

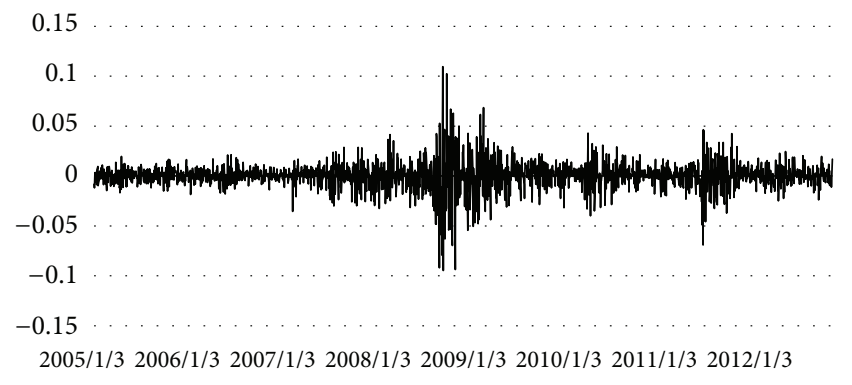

FIGURE 1: Logarithmic yield $x_{\mathrm{ROR}}(t)$ of S\&P 500 stock index.

4.1. Analysis of Multiple Structural Changes Model. The results of the multiple structural changes model for the initial index data are shown in Table 3.

The first issue to consider is to test for structural changes. The Sup $F_{T}(k)$ tests are all significant at the $5 \%$ level for $k=2,3,4,5$, except for the case $k=1$. The double maximum tests UDmax and WDmax, which allow us to test the null hypothesis of no structural break versus an unknown number of changes given the upper bound of five breaks, are significant at the $5 \%$ level. The significance of these tests does not provide enough information about the exact number of breaks but indicates that one break is at least present. We now turn to the $\operatorname{Sup} F_{T}(\ell+1 \mid \ell)$ tests, which are not significant for any $\ell \geq 3$ at the $5 \%$ level. The criteria BIC and LWZ choose four breaks for the series. Combined with all of the results, we obtain the conclusion that the optimal number of breaks is 4 .

According to the time series, the financial contagion period is divided into five stages. Those stages are as follows: the first stage is January 1, 2005, to September 25, 2006; the second phase is September 26, 2006, to September 12, 2008; the third stage is September 13, 2008, to November 23, 2009; the fourth phase is November 24, 2009, to February 1, 2011; and the fifth stage is February 2, 2011, to December 31, 2012. 
TABLE 4: Estimate results with the modified multiple structural changes model.

\begin{tabular}{|c|c|c|c|c|c|c|}
\hline \multicolumn{7}{|c|}{ Specifications } \\
\hline$z_{t}=\{1\}$ & $q=1$ & $p=0$ & $h=258$ & $M=5$ & $\varepsilon=0.15$ & $T=1720$ \\
\hline \multicolumn{7}{|c|}{ Tests } \\
\hline $\operatorname{SupF}_{T}(1)$ & $\operatorname{SupF}_{T}(2)$ & $\operatorname{SupF}_{T}(3)$ & $\operatorname{SupF}_{T}(4)$ & $\operatorname{SupF}_{T}(5)$ & $U D \max$ & WDmax \\
\hline 0.7920 & 1.3747 & 0.626 & 0.5655 & $5.1954^{*}$ & 5.1954 & 5.1954 \\
\hline $\operatorname{SupF}_{T}(2 \mid 1)$ & $\operatorname{SupF}_{T}(3 \mid 2)$ & $\operatorname{SupF}_{T}(4 \mid 3)$ & $\operatorname{SupF}_{T}(5 \mid 4)$ & & & \\
\hline 0.6364 & 0.6063 & 0.6038 & 0 & & & \\
\hline \multicolumn{7}{|c|}{ Number of breaks selected } \\
\hline Number of breaks & 0 & 1 & 2 & 3 & 4 & 5 \\
\hline $\mathrm{BIC}$ & -2.3577 & -2.4653 & -2.5376 & -2.6155 & -2.6994 & -2.6781 \\
\hline LWZ & -2.3571 & -2.4486 & -2.5048 & -2.5666 & -2.6345 & -2.597 \\
\hline \multicolumn{7}{|c|}{ Estimates with three breaks } \\
\hline$\widehat{\delta}_{1}$ & $\widehat{\delta}_{2}$ & $\widehat{\delta}_{3}$ & $\widehat{\delta}_{4}$ & $\widehat{\delta}_{5}$ & & \\
\hline 1241.55 & 1415.60 & 913.07 & 1169.42 & 1325.19 & & \\
\hline$(0.0366)$ & $(0.5211)$ & $(0.2213)$ & $(0.2971)$ & $(0.1533)$ & & \\
\hline$\widehat{T}_{1}$ & $\widehat{T}_{2}$ & $\widehat{T}_{3}$ & $\widehat{T}_{4}$ & & & \\
\hline $2006 / 10 / 9$ & 2008/9/11 & 2009/9/18 & $2011 / 5 / 18$ & & & \\
\hline
\end{tabular}

${ }^{*}$ Significance at the $5 \%$ level.

4.2. Analysis of Modified Multiple Structural Changes Model. We use the C-C method to reconstruct the phase spaces of the S\&P 500 stock index during the global financial crisis in 2008 and obtain the time lag $\tau=3$ and the embedding dimension $m=20$. We calculate that the dimension of the attractor $D$ is 2.2668 by the $G-P$ method; therefore, the time window $n n>10^{D}=184$. This paper uses the window width $n n=300$. The estimate results with the modified model are shown in Table 4

When $k=5$, the Sup $F_{T}(k)$ test is significant at the $5 \%$ level. Therefore, although we do not know the exact number of breaks, we can ensure the existence of breakpoints. The Sup $F_{T}(\ell+1 \mid \ell)$ tests and the double maximum tests UDmax and WDmax did not pass the test. The criteria BIC and LWZ are presented to determine the optimal number of breakpoints and optimal interval principle.

The paper determines that the optimal number of breakpoints is 4 , and the location of the breakpoint is given in Table 4. Therefore, the global financial crisis in 2008 is divided into five stages: the first stage is January 1,2005 , to October 9, 2006; the second stage is October 10, 2006, to September 11, 2008; the third stage is September 12, 2008, to September 18, 2009; the fourth stage is September 19, 2009, to May 18, 2011; and the fifth stage is May 19, 2011, to December 31, 2012. Figure 2 shows the results of stage division during the global financial crisis in 2008 based on the original multiple structural changes model and the modified multiple structural changes model based on the largest Lyapunov exponents. Considering the fluctuation of the data and the actual situation of the global financial crisis in 2008, the results based on the modified model are superior to those based on the original model. Based on the research from former scholars and the analysis results of our paper on the global financial crisis in 2008, we give some explanations for each stage. The first stage (January 1, 2005, to October

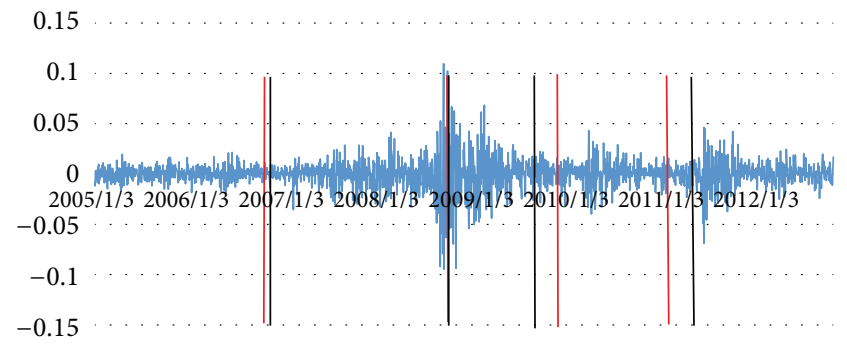

FIGURE 2: Results of stage division comparing the original model with the modified model.

$9,2006)$ is the stationary phase of financial crisis, when the global financial system experiences a Bullish market. The second stage (October 10, 2006, to September 11, 2008) is the local outbreak period of financial crisis. The outbreak only occurs in the US financial system during this time, and the global financial system has not been greatly affected. The third stage (September 12, 2008, to September 18, 2009) is the global outbreak and crisis contagion period. With the bankruptcy and collapse of a range of investment banks and financial institutions on Wall Street in September 2008, the financial crisis begins to spiral out of control and the crisis of the American financial system spreads to Europe and other countries. During the fourth stage (September 19, 2009, to May 18, 2011), the global financial crisis becomes increasingly stable under the efforts of the government in countries around the world. In the last stage (May 19, 2011, to December 31, 2012), with the continuous expansion of rescue, the proportion of debt increases dramatically and exceeds payment ability in some countries; the European debt crisis is emerging gradually. 
TABLE 5: The largest Lyapunov exponents and standard deviations for all-time series and various stages.

\begin{tabular}{|c|c|c|c|c|c|c|}
\hline & All-time series & First stage & Second stage & Third stage & Fourth stage & Fifth stage \\
\hline The largest Lyapunov exponents & 0.0707 & -0.0042 & 0.1694 & -0.4556 & -0.0129 & -0.2726 \\
\hline S.D. & 0.0139 & 0.0066 & 0.0111 & 0.0281 & 0.0104 & 0.0127 \\
\hline
\end{tabular}

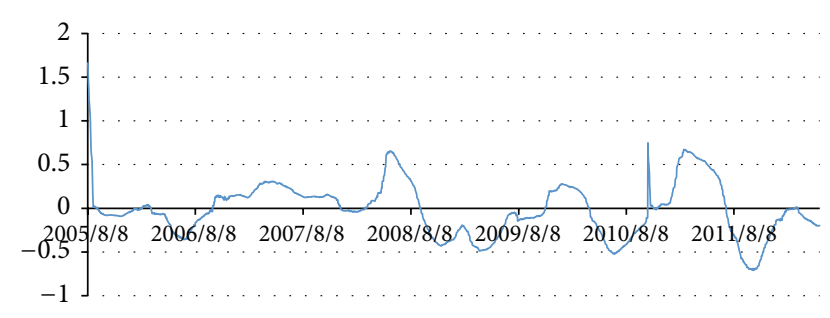

FIGURE 3: Variation of the largest Lyapunov exponents for all-time series.

During the analysis of the modified multiple structural changes model based on the largest Lyapunov exponents, we calculate 1720 largest Lyapunov exponents under a slip window for all-time series. Figure 3 shows the variation of the largest Lyapunov exponents for all of the time series. Table 5 shows the largest Lyapunov exponents and standard deviations for time series and various stages. The largest Lyapunov exponents are 0.0707 for all-time series, indicating that the financial system embodies obvious chaos characteristic and can be analyzed with a nonlinear model. There are significant differences in the largest Lyapunov exponents and standard deviations at various stages of financial crisis; the second stage has obvious chaos characteristics.

\section{Conclusions}

Since the 1990s, the nonlinear characteristic of the financial market has attracted more attention. In this paper, we have proposed a modified multiple structural changes model to test structural breaks of a financial system based on the largest Lyapunov exponents instead of relying on the index and correlation approach. We treat the breakpoints as unknowns and use the methods of Bai and Perron $[8,13]$ to estimate them. We use the Lorenz system as an example of a simulation experiment about the modified multiple structural changes model. The results show that the modified model has a very good judgment of breakpoint and could accurately reveal the change of nonlinear characteristics of a time series. Using daily data on the S\&P 500 stock index during the global financial crisis in 2008, we compare the results of the original multiple structural changes model and the modified model. Our empirical results show structural breaks in the largest Lyapunov exponents. We divide the financial crisis into four stages: stationary, local outbreak, global outbreak or crisis contagion, and recovery period. Considering the fluctuation of the data and the actual situation of the global financial crisis in 2008, the results based on the modified model are superior to those based on the original model. We also find that there are significant differences in the largest Lyapunov exponents and the standard deviations at various stages of financial crisis; in particular, the local outbreak period demonstrates obvious chaos characteristics.

\section{Conflict of Interests}

The authors declare that there is no conflict of interests regarding the publication of this paper.

\section{Acknowledgment}

This research is supported by the National Nature Science Foundation of China (nos. 71173060 and 71031003).

\section{References}

[1] M. Billio and L. Pelizzon, "Contagion and interdependence in stock markets: have they been misdiagnosed?" Journal of Economics and Business, vol. 55, no. 5-6, pp. 405-426, 2003.

[2] T. Gravelle, M. Kichian, and J. Morley, "Detecting shiftcontagion in currency and bond markets," Journal of International Economics, vol. 68, no. 2, pp. 409-423, 2006.

[3] P. Masson, "Contagion: macroeconomic models with multiple equilibria," Journal of International Money and Finance, vol. 18, no. 4, pp. 587-602, 1999.

[4] F. Kristin and R. Rigobon, "Contagion in Latin America: definition, measurement, and policy implications," NBER Working Paper 7885, 2001.

[5] R. Rigobon, "On the measurement of international propagation of shocks: is the transmission stable?" Journal of International Economics, vol. 61, pp. 261-283, 2003.

[6] G. M. Caporale, A. Cipollini, and N. Spagnolo, "Testing for contagion: a conditional correlation analysis," Journal of Empirical Finance, vol. 12, no. 3, pp. 476-489, 2005.

[7] M. McAleer and J. C. W. Nam, "Testing for contagion in ASEAN exchange rates," Mathematics and Computers in Simulation, vol. 68, no. 5-6, pp. 519-527, 2005.

[8] J. Bai and P. Perron, "Estimating and testing linear models with multiple structural changes," Econometrica, vol. 66, no. 1, pp. 4778, 1998.

[9] E. Essaadi, J. Jouini, and W. Khallouli, "The Asian crisis contagion: a dynamic correlation approach analysis," Panoeconomicus, vol. 56, no. 2, pp. 241-260, 2009.

[10] I.-M. Baek and J. Jun, “Testing contagion of the 1997-98 crisis in Asian stock markets with structural breaks and incubation periods," Journal of Asian Economics, vol. 22, no. 5, pp. 356-368, 2011.

[11] D. Hsieh, "Chaos and nonlinear dynamics: application to financial markets," The Journal of Finance, vol. 46, pp. 1839-1877, 1991.

[12] K. P. Lim and K. S. Liew, "Testing for nonlinearity in ASEAN financial markets," Working Paper, 2003.

[13] J. Bai and P. Perron, "Computation and analysis of multiple structural change models," Journal of Applied Econometrics, vol. 18, no. 1, pp. 1-22, 2003. 
[14] M. T. Rosenstein, J. J. Collins, and C. J. de Luca, "A practical method for calculating largest Lyapunov exponents from small data sets," Physica D: Nonlinear Phenomena, vol. 65, no. 1-2, pp. 117-134, 1993.

[15] J. Bai and P. Perron, "Multiple structural change models: a simulation analysis: a simulation analysis," in Econometric Theory and Practice: Frontiers of Analysis and Applied Research, D. Corbea, S. Durlauf, and B. E. Hansen, Eds., pp. 212-237, Cambridge University Press, Cambridge, UK, 2006.

[16] J. Bai and P. Perron, "Critical values for multiple structural change tests," The Econometrics Journal, vol. 6, no. 1, pp. 72-78, 2003.

[17] A. Wolf, J. B. Swift, H. L. Swinney, and J. A. Vastano, "Determining Lyapunov exponents from a time series," Physica D: Nonlinear Phenomena, vol. 16, no. 3, pp. 285-317, 1985.

[18] J. Geweke and S. Porter-Hudak, "The estimation and application of long memory time series models," Journal of Time Series Analysis, vol. 4, no. 4, pp. 221-238, 1983. 


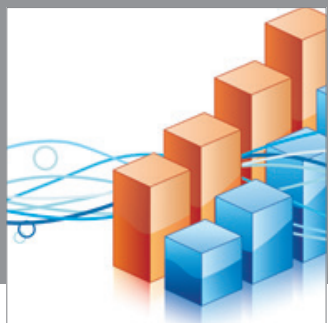

Advances in

Operations Research

mansans

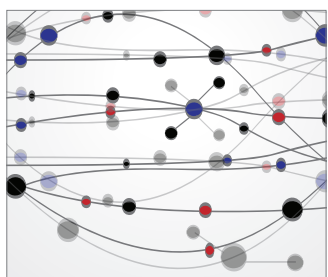

The Scientific World Journal
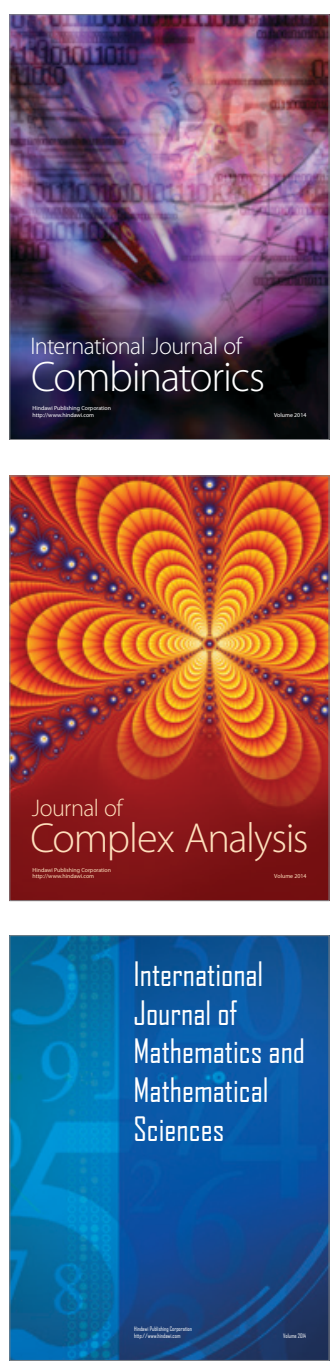
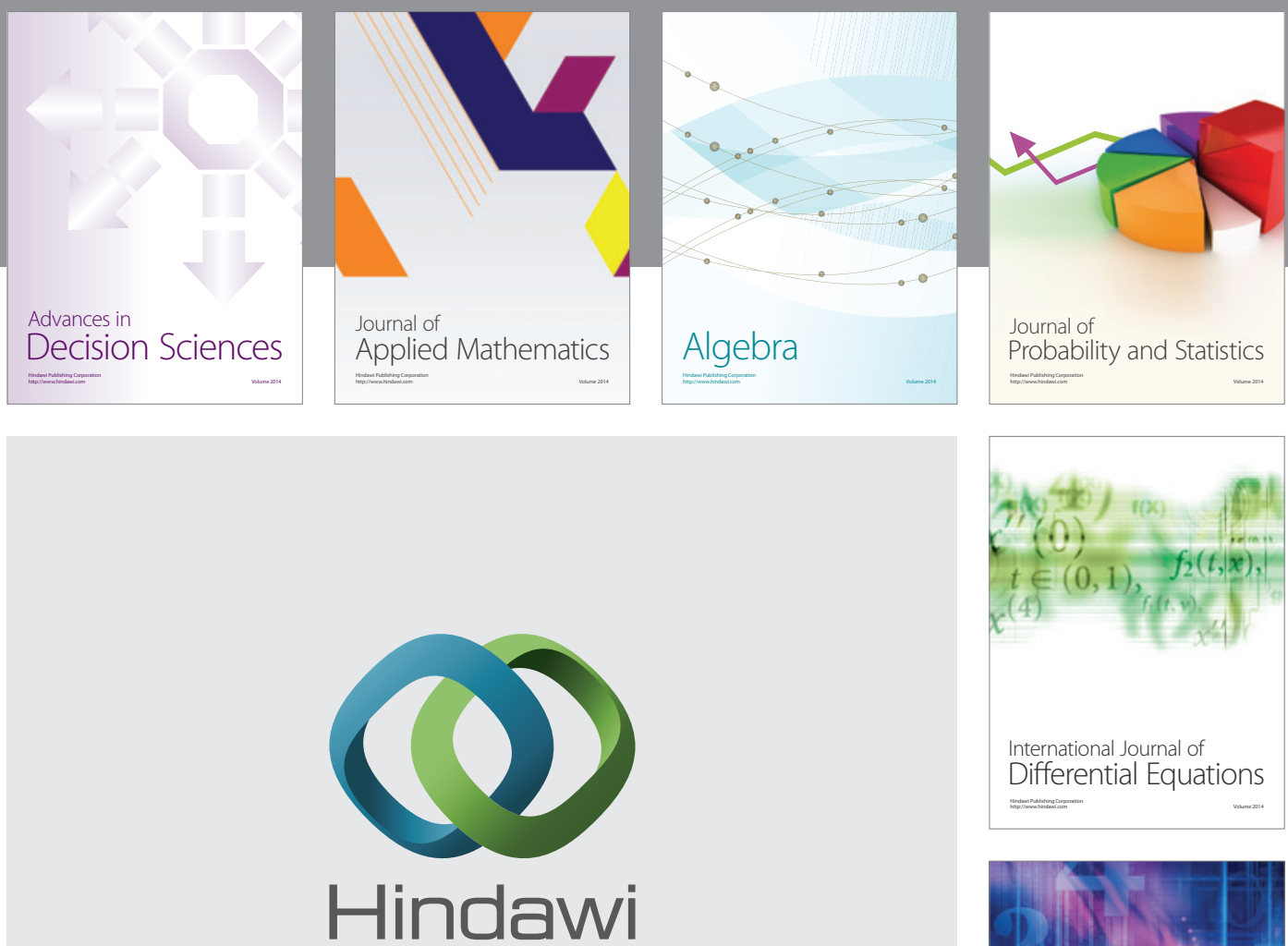

Submit your manuscripts at http://www.hindawi.com
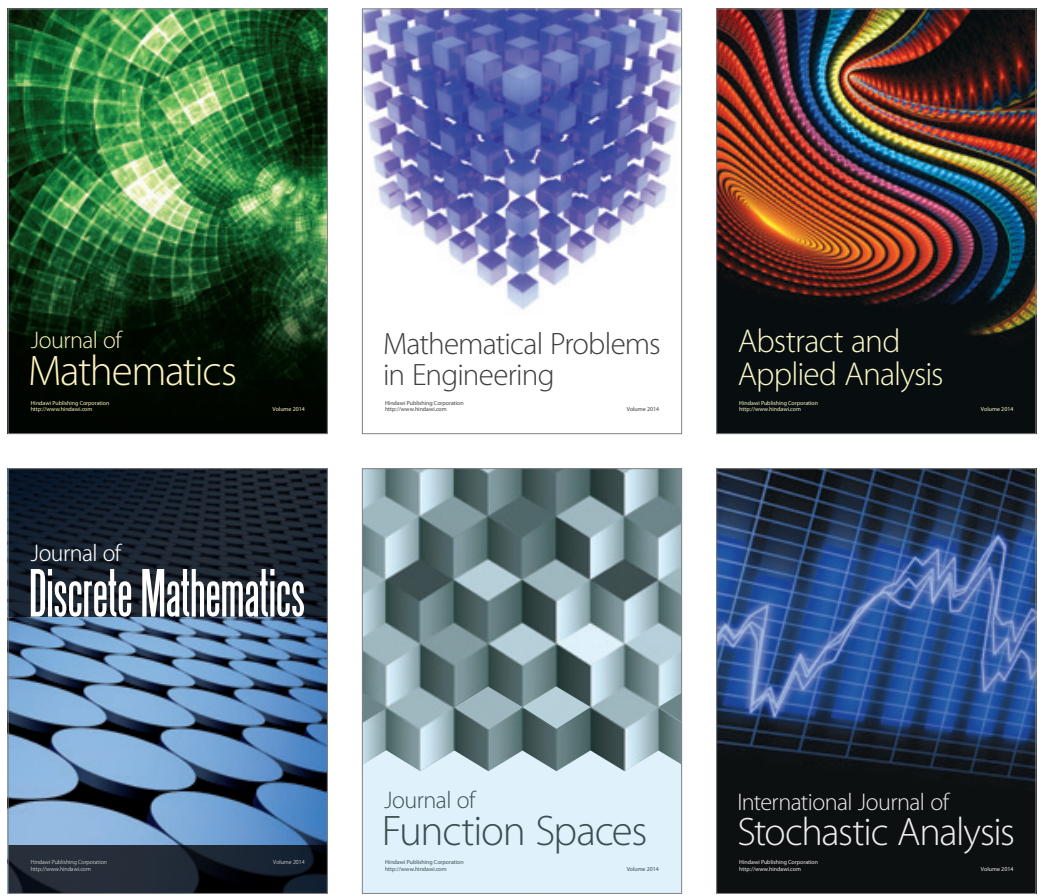

Journal of

Function Spaces

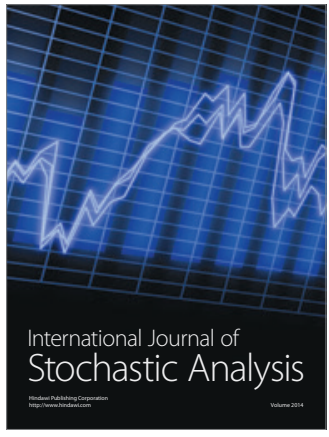

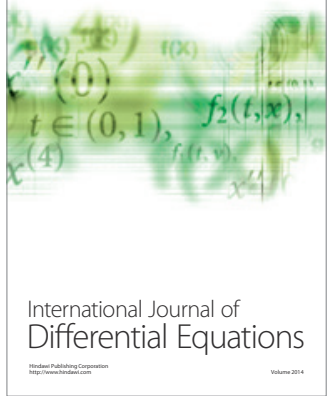
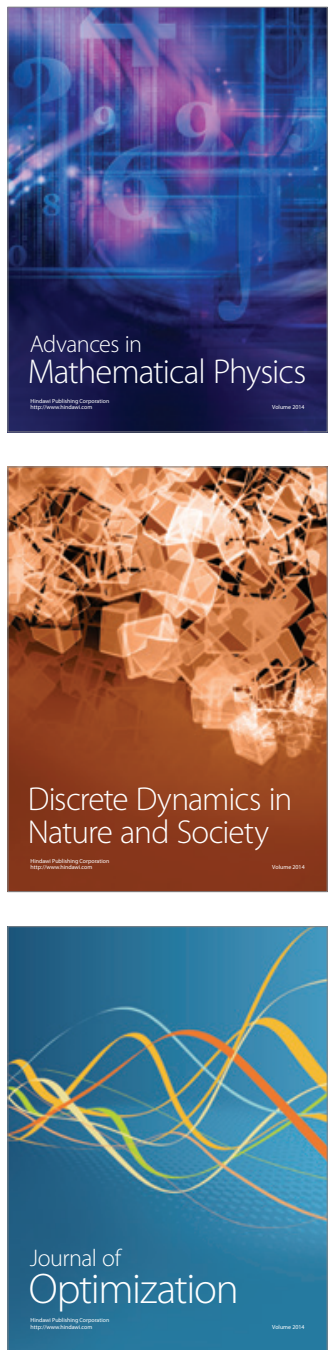